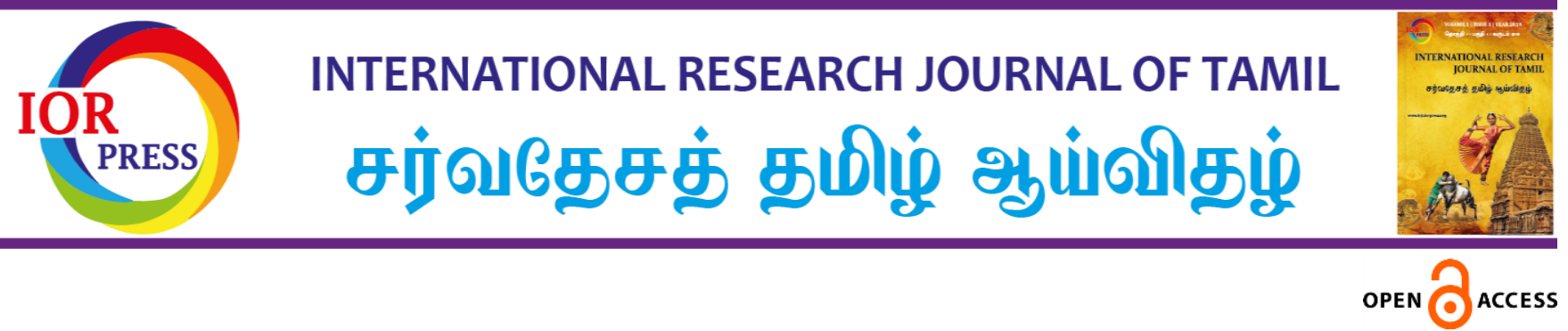

மார்க்சிய இலக்கியக் கோட்பாடுகள், ோஷலிச யதார்த்தவாதம் வழி சுரங்கம் புதின ஆய்வு

இரா. குமரகுருபரன் அ, *

அ தமிழியல் மற்றும் பண்பாட்டுப் புலம், தமிழ்நாடு திறந்தநிலைப் பல்கலைக்கழகம், சென்னை-600015, தமிழ்நாடு, இந்தியா.

\title{
A Study of Marxist Literary Theories and the Novel Surangam in a Socialist Realist Way
}

\section{R. Kumaraguruparan ${ }^{a}$,}

a School of Tamil and Cultural Studies, Tamil Nadu Open University, Chennai-600015, Tamil Nadu, India

* Corresponding Author: vinci.illustrator@gmail.com

Received: 25-03-2021

Revised: 03-02-2022

Accepted: 05-02-2022

Published: 28-02-2022

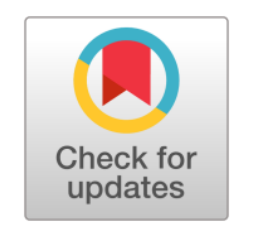

\begin{abstract}
What led Prof Prabhat Patnaik, Pope at Vatican City and Dr Raghuram Rajan to a rallying point for the recovery and sustainability of the Global Capitalism from its shivering crisis? It's Karl Marx's 'Capital' ('Das Kapital'). Literature Review of Miners' plight in Indian Literature. World's top ten mine disasters in which two happened in India - Dhanbad and Chasnala. ' Kaala Pathar' Hindi film was based on Chasnala mining disaster. Nationalisation of Indian Coal Industry thanks to Indian coal miners' continuous struggles. Introduction of Ku.Chinnappa Bharathi, Tamil writer, who authored 'Surangam' Tamil novel. How did the idea of writing 'Surangam' come to Ku. Chinnappa Bharathi's mind? Shri Bikas Chowdhury, CPI-M MP from Asansol Constituency, invited the writer to pay a visit to Dhanbad, Jharia and Asansol mines, to study the plight and living standards of the workers to attempt a novel on this theme. KuChiBha. accepted the offer. The conversation with the miners with the help of interpreters, contributed a lot to visualize the air. Plot of Surangam. The category of workers and the hierarchy of supervisors. Bikas Choudhary, Jamuna Kumari, Sakuntala Devi, Mining Munshi, Mining Sardar, Mine owner Tiwari, organised workers, drunkardworkers, Debt recovery agents, liquor shop-owner, villagers, peasants and the like. The greed of inhuman mine-owners and supervisors is portrayed the customs and cultural events of Bengalis and the tenets of humanitarian Marxist Atheism are briefed. How the novel observes the genre of Socialist Realism, with the Marxist point of view, is wellpresented. The denationalisation of the Coal Industry, with the repeal of Mining Nationalisation Acts, will again unleash the exploitation of private owners in the coal industry. The case-study of ' Surangam ' to explore the livelihood of the miners in nonTamil speaking areas to write a novel in Tamil, for being translated further into Bengali and Hindi, is a laudable attempt and a great literary venture.
\end{abstract}

Keywords: Marxist Novel, Surangam, Chinnappa Bharathi, Socialist Realism

\section{முன்னுரை}

2008 -ஆம் ஆண்டு அமெரிக்கப் பொருளாதார வீழ்ச்சியைத் தொடர்ந்து உலகளாவிய நிதிநிலை மீட்சிக்காக ஜோசப் ஸ்டிக்லிட்ஸ் தலைமையில் ஐக்கிய நாடுகள் சபை அமைத்த வல்லுநர்கள் குழுவில் நால்வரில் ஒருவராக நியமிக்கப்பட்டவர் பேராசிரியர் பிரபாத் பட்நாயக். உலகளாவிய 
அளவில் போப்பாண்டவர் முதல் அனைத்து கிறிஸ்தவப் பாதிரிமார்களும் மார்க்ஸ் எழுதிய "மூலதனம்" நூலின் பிரதிகளில் என்னதான் கூறப்பட்டுள்ளது என்று அறியத்தலைப்பட்டதன் விளைவாக மூலதனம் நூல்கள் விற்பனை அதிகரித்தது. இதுவும் 2008-ல்தான். கார்ல் மார்க்ஸ் 150-வது ஆண்டு நினைவுநாள் அஞ்சலி ஒன்றில் முதலாளித்துவப் பொருளாதார அறிஞர் ரகுராம் ராஜன், கார்ல் மார்க்சின் பங்களிப்பை, அவரது கண்ணோட்டத்தில் அலசும் காணொளியில், பெரிதும் சிலாகிக்கப்படுகிறார் மார்க்ஸ்! மேற்கண்ட மூன்று நிகழ்ச்சிகளுக்கும் தொடர்பு உண்டு. அதுதான் மார்க்சியம். பிரபாத் பட்நாயக் இந்தியாவின் தலையாய மார்க்சியப் பொருளாதார அறிஞர் / சிந்தனையாளர். மார்க்சின் நாத்திகக்கருத்துகளால் (மார்க்ஸ் 1841- ஆவது ஆண்டில் பெற்ற முனைவர் பட்டம் "டெமாக்ரிடியத் தத்துவத்திற்கும் எபிக்யூரிய இயற்கைத் தத்துவத்திற்குமிடையிலான வேறுபாடு "குறித்தது. அதாவது, தத்துவத்திற்கு இறையியல் அடிபணிந்துதான் ஆகவேண்டும் என்பது இந்தக் கோட்பாட்டின் தாக்கம்.) அதிர்ந்து உறைந்துபோய் அவரை நிராகரித்த அப்போதைய வாட்டிகன் திருச்சபையின் செய்கைக்கு, 2008 - ஆவது ஆண்டில் 'பாவமன்னிப்பு' தேடக்கொண்டது கத்தோலிக்க திருச்சபை! உலகமயமாக்கல் காலகட்ட இந்தியாவின் பொருளாதாரச் சீர்திருத்தங்களை ஆதரித்த முதலாளித்துவப் பொருளாதார அறிஞரான ரகுராம் ராஜனும் நிராகரிக்க இயலாத அறிஞர் டாக்டர் கார்ல் மார்க்ஸ்!, எனவே மார்க்சியம் குறித்து அறிவது அவசியம். ஆழ்துளைக்கிணற்றில் தவறிவிழுந்த குழந்தைகள் குறித்த பதைபதைப்புச் செய்திகளைத் தொலைக்காட்சிகளில் காணும்போது துடிக்கும் நமது இதயங்களின் ஒலியும், மீட்கப்பட்டவுடன் எழும் நிம்மதிப்பெருமூச்சும், மீட்க இயலாது கைவிடப்பட்டபோது எழும் கேவலும் நமது உள்ளத்தைச் சிதைக்கும் என்பது கண்கூடு. இந்நிலையில், 'பூமித்தாயின் புத்திரர்களாக ' அன்றாடம் அவளது நிலத்தடி மடியில் பணியாற்றிவிட்டு வெளிவரும் சுரங்கத்தொழிலாளிகளின் அன்றாட அவலம் குறித்து அறிவோமா என்றால் இல்லை எனலாம். இத்தனைக்கும், தொழில் நுட்பத் தகவல்கள் இப்போது ஏராளம் உண்டு. ஆனால் இதை இலக்கியமாக்குவது ஒருசிலருக்கு மட்டுமே சாத்தியமாகிறது. இலக்கியத்தில் கதைகளாக, புதினமாக வெளிப்படுவதாகத் தேடினால் தெலுங்கு மொழியில் சிறுகதைகளும் ஒரு புதினமும் உண்டு. அதிலும் வாழ்வியலைவிட காதலே பிரதான களம். சாஸ்நளா சுரங்க விபத்தைக் களமாகக் கொண்டு "காலா பத்தர்" இந்தி திரைப்படம் 1979- ஆவது ஆண்டு வெளிவந்து நடிகர்கள் சத்ருகன் சின்ஹாவிற்கும் அமிதாப் பச்சனுக்கும் பெயர் - விருதுகள் வாங்கிக் கொடுத்ததோடு சுரங்க தொழிலாளர்கள் அவல வாழ்வையும் பேசவைத்தது.

மார்க்சியத் தத்துவத்தின் அடிப்படை, மார்க்சியத்தின் இயக்கவியல் கூறுகளான இயக்கவியல் பொருள் முதல் வாதம், வரலாற்றியல் பொருளாமுதல்வாதம், மார்க்சியம்- வரையறை, மார்க்சிய இலக்கியக் கோட்பாடுகள், அடித்தளம் - மேற்கட்டுமானம், பிரதிபலிப்புக் கொள்கை, யதார்த்தவாதம், சோஷலிசயதார்த்தவாதம், வரலாற்றுச் சூழமைவு ஆகிய மார்க்சியக் கோட்பாட்டுக் கூறுகளின் வெளிச்சத்தில் ஆராய்கிறது.

இப்புதினத்தை எழுதும் முன்பாக நிலக்கரிச் சுரங்கத் தொழிலாளர்கள் குறித்த புதினங்கள் இந்திய இலக்கியத்தில் வெளிவந்துள்ளதா என்பது குறித்து சுரங்கம் புதின ஆசிரியர் குசிபா. விசாரித்தறிய முனைகிறார். ஒன்றுபட்ட ஆந்திரப் பிரதேசத்தில்தான் (இப்போது தெலுங்கானா) சிங்கரேணி நிலக்கரிச்சுரங்கமுள்ளது. சுரங்கத்தைக் களமாகக்கொண்டு தெலுங்கில் சிறுகதைகள் வந்துள்ளன நாவல் ஒன்று வெளியாகியிருப்பதாகவும் என்று அவர் கேள்வியுற, 'ஸ்தாபன ரீதியாக ஒன்றுபட்ட தொழிலாளர்களின் போராட்ட எழுச்சியை முன்னெடுத்துச் செல்லும் புரட்சித்தன்மை கொண்டதா? ' என்று மேலும் வினவுகிறார். 'இல்லை. வாழ்வும், ரொமான்சும் நிறைந்த படைப்பாக நாவல் ஒன்று வந்துள்ளது' என்கிறார்கள்." எனவே நாவலின் குணாம்சம் தனித்துவம் மிக்கதாகவே இருக்கும் என்று ஆறுதல் பெற உதவியது. அந்த வகையில் கர்வபங்கம் நிகழாமல் தப்பித்தேன்" என்று முன்னுரையில் இதைக்கூறுகிறார்.

\section{இந்தியாவில் நிலக்கரிச் சுரங்கத்தொழில்}

இந்தியாவின் நிலக்கரிச்சுரங்கத் தொழில் 1774-ஆவது ஆண்டில் தனியாரால் தொடங்கப்பட்டது. உலகிலேயே துரிதமாகச் செயல்படும் சுரங்கங்களில் இரண்டாவது இடம் இந்தியாவிற்கே. 
உலோகவியல் நிலக்கரி / அல்லாத நிலக்கரி ஆகிய இருவகையும் இரும்பு எஃகு தொழிற்சாலைகள், மின்சாரம், கனரகத் தொழிற்சாலைகள், இரயில்வே துறை மற்றும் இதர கேந்திரியத் தொழில்களுக்கு அன்றாடம் தேவைப்படுபவை. ஆனால், இவற்றை உருவாக்கும் நிலக்கரிச் சுரங்கத் தொழிலின் ஆபத்தான பாதுகாப்புச் சூழலும் தொழிலாளர்களின் பணிநிலைமைகளும் இன்னும் சரியாகப் பலராலும் உணரப்படவில்லை. உலகன் மிகப்பெரும் பத்து சுரங்க விபத்துகளில் இரண்டு இந்தியாவில் நிகழ்ந்துள்ளன. தன்பாத் (ஜார்கண்ட்) தோரி நிலக்கரிச் சுரங்கத்தில் 28 மே 1965-ஆம் ஆண்டு நிகழ்ந்த தீ விபத்தைத் தொடர்ந்து சுரங்கம் வெடித்துச்சிதறி 268 தொழிலாளர்கள் உயிரிழந்தனர். இந்தச் சுரங்கம் தனியாரான ராஜா ராம்கருக்குச் சொந்தமானது. இந்த விபத்தின் எதிர்வினையாக, தனியார் நிலக்கரிச் சுரங்கங்களை அரசே ஏற்று நடத்த வேண்டும் என்ற தொழிலாளர்களின் போராட்டங்கள் வலுத்தன. இந்நிலையில், மத்திய அரசு 1971-72 ஆம் ஆண்டில் சுரங்கங்களை நாட்டுடைமை ஆக்கியது. உலோகவியல் நிலக்கரிச் சுரங்க தேசியமயமாக்கல் சட்டம் - 1972 நிலக்கரிச் சுரங்கம் (தேசியமயமாக்கல்) சட்டம் - 1973 ஆகியன நிறைவேற்றப்பட்டன. அடுத்ததாக, 27 டிசம்பர், 1975 சாஸ்நளா (ஜார்கண்ட்) நிலக்கரிச் சுரங்கம் வெடித்து அதன் உடனடி விபத்தாக 32,000 கியூபிக் மில்லியன் தண்ணீர் உட்புகுந்த வெள்ளப்பெருக்கில் 380 சுரங்கத் தொழிலாளர்கள் (இதில் 130 ஒப்பந்த ஊழியர்கள்) சிக்கிப் புதைந்து மாண்டனர். சுரங்கம் பொதுத்துறை இஸ்கோவுக்குச் சொந்தமானது நிறுவனம் உலகத்தரத்தில் உள்ளதாகச்சொல்லித் தப்பித்ததை எதிர்த்து வழக்கு நடைபெற்றது. நாற்பது ஆண்டுகள் கழித்து 2012 -ல் விபத்து குறித்த தீர்ப்பு வெளியானது. கவனக்குறைவினால் நடைபெற்ற விபத்து என்று குற்றம் சாட்டப்பட்ட இருவருக்கு ஓராண்டுச் சிறையும், ஆளுக்கு ரூபாய் 5000 அபராதமும் விதிக்கப்பட்டன.

\section{எழுத்தாளர் கு. சின்னப்பபாரதி}

"போராளியாகவும் படைப்பாளியாகவும் தமிழுக்குக் கிடைத்திருப்பவர்கள் மிகக்குறைவு. அப்படிப்பட்டவர்களில் முக்கியமான ஒருவர் கு. சின்னப்பபாரதி. போர்க்குணம் மிக்க மனிதாபிமானி அவர். படைப்பையும் வாழ்வையும் வேறுபடுத்திப் பார்க்க முடியாத நேர்மையாளர். இலக்கியத்தை வெறும் தொழில்நுட்பத் திறமையாகவோ, கற்பனை வடிவமாகவோ பார்க்காமல் சமூக வாழ்வின் நேர்மையான வெளிப்பாடாகப் பார்க்கிற அழுத்தமான எதார்த்தவாதி.

ஜீவா பெரும் போராளி. ஆனால் வாழ்வு முழுவதும் தீவிரப் படைப்பாளியாயிருந்தவரல்ல. ஜெயகாந்தன் தொடக்க காலத்தில் ஓரளவு அரசியல் சார்புள்ள படைப்பாளி. ஆனால் அவருடைய இயக்க அனுபவத்துக்கும் சின்னப்பபாரதியின் இயக்க அனுபவத்துக்கும் வேறுபாடு அதிகம். ரகுநாதன் கூட கு.சி.பா. போல நேரடியான இடதுசாரிப் போராட்ட அனுபவம் பெற்றவர் அல்ல. சொல்லப்போனால் சின்னப்பபாரதி அளவுக்கு இயக்கப் போராட்ட அனுபவம் பெற்ற இலக்கியவாதி இன்றைய தமிழகத்தில் வேறுயாரும் இல்லையென்றே தோன்றுகிறது."

என்று தமிழ்நாடு கலை இலக்கியப் பெருமன்றத்தின் நிர்வாகியும் சாகித்திய அகாதெமி விருதுபெற்ற எழுத்தாளருமான பொன்னீலன், கு. சின்னப்பபாரதி குறித்து எழுதியிருக்கும் மேற்கோள் புத்தகப் பின்னட்டையில் இடம்பெறுகிறது.

'சரஸ்வதி காலம்' எனப்படும் 'சரஸ்வதி' சிற்றிதழ் தொடங்கிய காலம் முதலாகவே 1950களிலிருந்து - சிறுகதைகளையும் கட்டுரைகளையும் கவிதைகளையும் எழுதி வருபவர் 'குசிபா.' என்று அழைக்கப்படும் எழுத்தாளர் கு. சின்னப்ப பாரதி ஆவார். ஜனசக்தி, தீக்கதிர் முதலான நாளிதழ்களிலும் தாமரை, செம்மலர், சிகரம் முதலான மாத இதழ்களிலும் இவரது படைப்புகள் வெளியாகி வாசகர்களிடையே பெரும் வரவேற்பைப் பெற்றிருக்கின்றன. 'சங்கம்' புதினம் 1986 இலக்கியச் சிந்தனை விருது பெற்றது. இந்திய கம்யூனிஸ்ட் கட்சியின் (மார்க்சிஸ்ட்) இலக்கிய ஏடான "செம்மலர்" மாத இதழில் தாகம், சங்கம், சர்க்கரை உள்ளிட்ட படைப்புகள் தொடராக வெளிவந்தன.

தமிழ்நாடு முற்போக்கு எழுத்தாளர் கலைஞர்கள் சங்கத்தின் தலைமைப் பொறுப்பில் இ இருந்த அனுபவம் மிக்கவர் குசிபா. தமுஎகச / குப்பண்ணன் - பெருமாயி விருது இவரது பெற்றோர் நினைவாக 
இவரால் ஏற்படுத்தப்பட்டது. அவரது' கு. சின்னப்ப பாரதி இலக்கிய அறக்கட்டளை 'ஆண்டுதோறும் சிறந்த படைப்புகளுக்கு விருதுகள் வழங்கிச் சிறப்புச்செய்து வருவதும் குறிப்பிடத்தக்கது. தாகம், சங்கம், சர்க்கரை, பவளாயி, தலைமுறை மாற்றம் முதலான நாவல்கள் ஆங்கிலம், இந்தி, வங்கம், குஜராத்தி, தெலுங்கு, மலையாளம் கன்னடம், மராட்டி, பிரெஞ்சு ஆகிய மொழிகளில் மொழிபெயர்க்கப்பட்டுள்ளன. 'சுரங்கம்' புதினம் உபாலி நாணயக்காரவின் மொழிபெயர்ப்பில் சிங்கள மொழியில் வெளியாகியுள்ளது குறிப்பிடத்தக்கது

\section{'சுரங்கம்' எழுதப் பின்புலம்}

மேற்கு வங்கத்தின் தலைநகரான கொல்கத்தாவிலுள்ள இந்திய கம்யூனிஸ்ட் கட்சியின் (மார்க்சிஸ்ட்) புரமோத் தாஸ்குப்தா மையத்தில் கு.சி.பா. 1998- ஆம் ஆண்டு தங்கியிருந்தபோது, இந்திரநாத் பானர்ஜி மொழிபெயர்த்திருந்த ஆங்கில மொழிபெயர்ப்பு மூலம் தன்னை அறிந்திருந்த அசன்சால் நாடாளுமன்ற மக்களவை உறுப்பினரான பிகாஸ் சௌதரியைச் சந்தித்தார். அவரது வேண்டுகோளை ஏற்று நிலக்கரிச் சுரங்கத் தொழிலாளர்களின் வாழ்க்கையைப் பற்றி நாவல் எழுதும் முயற்சியைத் தொடங்கினார். தத்துவ நோக்கமும், கற்றறிதலின் அனுபவத் தேலும் ஒன்றுபட்டுவிட்டால் சுலபத்தில் எழுதிவிடலாம் அதற்கான வசதிகள் செய்து கொடுக்கிறேன்' என்று பிகாஸ் சௌதரி உறுதிகூறியதோடு மட்டுமல்லாமல் அவரது போராட்ட அனுபவங்களையும் பகிர்ந்தபடியே ஊக்குவித்ததனால் இந்த நாவல் சாத்தியமானது என்கிறார் குசிபா., இதன் முன்னுரையில்.

\section{கள ஆய்விற்கு உதவியோர்}

பிகாஸ் சௌதரி வழிகாட்டலில் தன்பாத், ஜாரியா, அசன்சால் பகுதிகளிலுள்ள நிலக்கரிச் சுரங்கத் தொழிலாளர்களிடையே தங்கி எண்ணூறு அடி முதல் ஆயிரத்து நூறு அடி ஆழம் வரை கொண்ட சுரங்கங்களுக்குள் சென்று தொழில் நுணுக்கங்களையும், தொழிலாளர்கள் படும் சிரமங்களையும், வேதனைகளையும் கற்றறிந்து மொழிபெயர்ப்பாளரும் எழுத்தாளருமான தமது நண்பர் கல்கத்தா கிருஷ்ணமூர்த்தி துணையுடன் வங்க, இந்தித் தொழிலாளர்களிடம் பேசிப்பழகி விவாதித்து, அவர்களின் வாழ்க்கைப்பாடுகளை அறிந்து, புதினமாகப் படைத்துள்ளார் கு.சி.பா (Chinnappa Bharathi, 2012).

"என் மண் சார்ந்ததல்லாத ஒரு பிரச்சினையை நான் எடுத்துக் கொண்டதன் மூலம் எனது மற்ற நாவல்களைப்போல் வெற்றிகரமாக உருவாக்க முடியவில்லை என்பதை உணர்கிறேன். ஆனால் மனித வாழ்வின் சிரமங்களும் பிரச்சினைகளும் எங்கும் ஒரேவகைப்பட்டதானதால் மனிதர்களைப் புரிந்து கொள்வதிலான தடைகள் இடையூறுகளாக அமையவில்லை என்பது சாதகமான விஷயம். இருப்பினும் அம் மக்களின் முழு ஆன்மாவையும் தரிசிப்பதென்பது சாத்தியமற்ற விஷயம். அம் மனிதர்களுடன் கைபிடித்து, அம்மனிதர்களுக்குள் உருவாகும் அகமனப் போராட்டத்தை வெளிப்படுத்துவதும் கடினமான ஒன்று. இச் சிரமங்களுக்கு மத்தியில்தான் அவர்களின் கரங்களைப் பற்றவும் ஆன்மாவை தரிசிக்கவும் முயன்றிருக்கிறேன்." என்று மிகுந்த தன்னடக்கத்துடன் முன்னுரையில் குறிப்பிடுகிறார்.

இந்நாவல் வெளியிடத் தூண்டிய பிகாஸ் சௌதரி, நாவல் வெளியான காலத்தில் (2006) இதைக்கண்ணுற இயலாது, ஆகஸ்டு 2005 ஆம் ஆண்டிலேயே மறைந்து விட்டது மிகவும் சோகமானது. புதினத்தின் முதன்மை மாந்தர் பிகாஸ் சவுதரி மூலமாக நிலைத்து வாழ்கிறார்.

\section{'சுரங்கம்' கதைச் சுருக்கம்}

வேலை, கூலி, உயிர்ப் பாதுகாப்பும் உத்தரவாதமும் அற்ற நிலக்கரிச் சுரங்கத் தொழிலாளர்கள், இழப்பதற்கு ஏதுமின்றி, தங்களது உழைப்புச்சக்தியை முதலாளிகளின் லாபத்திற்காக மட்டுமே விரயப்படுத்தி உழல்கின்றனர் முதலாளிமார்களின் கஜானாவை நிரப்புகிறார்கள். சிறு தவறு என்று சொல்லி நியாயமான கூலியையும் சுரண்டிக் கொழுக்கிறார்கள் மேலதிகாரிகள். இதைக்களைய சங்கம் 
அமைத்துப் போராடத் தங்களுள் ஒருவனான பிகாஸ் சௌதரியிடம் தலைமைப்பண்பு இருப்பதை சோதனைக்காலங்களில் உணர்கிறார்கள். பிகாஸ் குண்டர்களால் தாக்கப்படுகிறான். இதனால் போராட்டம் தொடர்கிறது. பண்ணையடிமைகளாக இருந்தபோதுள்ள அனுபவ அச்சம் போராட்டப்பாதை ஊடாகக் குறுக்கிட்டுத் தடைசெய்கிறது.

'எப்படியாவது காலம் மாறிவிடும், காளி கண்திறப்பாள்' என்ற நம்பிக்கையுடன் இருந்த பல தொழிலாளர்கள் இப்போது நிலைமையை உணர்ந்து தொழிற்சங்க உணர்வோடு வேலைநிறுத்தத்தில் இறங்கி, நிலக்கரிச் சுரங்கத் தொழில் நாட்டுடைமையாக்கப்பட அணிதிரள்கிறார்கள். சுரங்கம் அமைக்கத் தங்களது விளைச்சல் நிலங்களை இழப்பீடு பெறாமல் விட்டுக்கொடுத்து எதிர்பார்ப்பில் இருந்த விவசாயிகளையும் தனி அமைப்பின் கீழ் இணைத்துக்கொண்டு போராட்டம் நடத்த அறைகூவல் விடுக்கிறான் பிகாஸ். மாவட்ட ஆட்சியரிடம் விண்ணப்பம், உண்ணாவிரதம், முற்றுகை, சிறை என்று பலவழிகளில் போராட்டப்பாதை தீர்மானிக்கப் படுகிறது.

ஒருவாறாக, நிலக்கரிச் சுரங்கம் நாட்டுடைமையாக்கப்பட்டு விட்டது. ஆனால், காட்சி மாறினாலும் ஆட்சி மாறவில்லை! முதலாளி அகற்றப்பட்டு அதற்குப் பதிலாக, அரசுத்தலைமைப் பொறுப்பு, தனி அமைச்சர், பெர்சனல் மேனேஜர், ஜெனரல் மேனேஜர் என்று நிர்வாக அமைப்பின் பெயர்களில் மட்டுமே மாற்றம் கூலி கூடினாலும், நிலக்கரி வெட்டி எடுப்பதில் அதே நடைமுறையான முறையற்ற சுரண்டல் தொடர்கிறது. தொழிற்சங்கத்தலைவராக பிகாஸ் பலமுறை எச்சரித்தும் சுரங்கக் கட்டமைப்பு சரிசெய்யப்படாத நிலையில், நிர்வாகத்தின் அலட்சியம் காரணமாக விபத்து நேர்ந்து, பல தொழிலாளர்கள் பலியாகின்றனர். சகலபகுதித் தொழிலாளர்களும் உயிர் நீத்தோரின் நினைவாகத் திரண்டெழுப்பிய அஞ்சலி கீதம் எதிர்கால சமத்துவ வளவாழ்வுக்கான எழுச்சி கீதமாகிறது.

\section{யதார்த்த சூழல் சித்திரிப்பு}

நகமும் சதையுமான மனிதர்கள் எலிகளை விடக் கேவலமான வாழ்விடங்களில் கறையான் புற்றுப் போன்ற குடிசைகளில் குடும்பம் நடத்துகின்றனர். கறையான் புற்றுக்கு பாம்புகள் வருவது வழக்கம்தானே? விஷப்பாம்புக்கடிக்கு இரையாகும் மக்களும் உண்டு. பன்றிகள், நாய்கள் படுத்த கெட்ட வாடை, 'பகலில் உட்கார உதவும் கயிற்றுக் கட்டில், இரவில் குழந்தைகளிடமிருந்து கணவன் மனைவியின் அந்தரங்கம் காக்கும் தடுப்புச்சுவராக எழுந்து நிற்கும். நெருக்கடியின் நிர்ப்பந்தம் மனிதர்களுக்குண்டான வெட்க உணர்வுகளைக்கூடத் துடைந்தெறிந்துவிடுகிறது போலும். "ஆறுமணிக்கு காலைச்சங்கு ஒலித்ததும் கூண்டிலிருந்து சர்க்கஸ் வளையத்துக்குள் நுழையும் மிருகங்களைப்போல் சுரங்கத்துக்குள் நுழையும் தொழிலாளர்கள், கறையான் புற்றிலிருந்து ஈசல்கள் இறக்கை முளைத்துப் பறப்பது போல லிப்டிலிருந்து வெளியேறுகிறார்கள். மேல்பகுதி இயந்திரக் கொட்டகையில் 'பிட்பாட்டத்தில்' (உள் அடித்தளம்) இருப்பவர்களுக்குக் கேட்குமாறு மின்சார மணியொலிப்பு பணிநேரம் முடிவடைவதைத் தெரிவிப்பதும், விளக்கையும் ஹெல்மெட்டையும் தொப்பியையும் காவலாளியிடம் ஒப்படைத்துக் கையெழுத்திட்டு தொழிலாளர்கள் கீழிருந்து மேலாக வெளியேறுவதும், அடுத்த 'ஷிப்ட்' தொழிலாளர்கள் மேலிருந்து கீழிறங்கி அவசர அவசரமாக உள்வேலைக்கான உடைகளை மாற்றிக் கொண்டு 'கேப் லேம்ப' அறைக்குச் செல்வதும், 'ஹெல்மெட்-ஹெட்லைட்-பேட்டரி' அணிகலன்களை இடுப்பில் இறுக்கமாகக் கட்டிக் கொள்வதும் எந்திரத்தனமாக நடைபெறுகின்றன. தண்டா (மூங்கில் கழி) கொண்டு சுவற்றைத் தட்டிப்பார்த்து சுவர்க்கூரை வலுவை சோதிப்பது, 'சேப்டி லேம்ப்' மூலம் 'மீதேன் கேஸ், கார்பன்-டை- ஆக்ஸைடு' கசிவு சோதிப்பது' மைனிங் சர்தார்' கடமை. நீர்க்கசிவு உடைப்பு சோதனை, நீர் எந்திரம் செயல்பாட்டைக் கண்காணிப்பது 'பம்ப் கலாசி' யின் பணி. நிலக்கரிப் படிவத்தை லேசான வெடிவைத்துத் தகர்க்கும் 'டிரில்லர்' உடைபட்ட கரியை வெட்டிக் குவிப்பவர் அதை இரும்புத் தொட்டியில் அள்ளிப்போட்டுப் பாரம் ஏற்றுபவர்கள் என்று மூவகைப் பிரிவினர் இங்கு உடலுழைப்புத் தொழிலாளர்கள்.

கிணற்றுத்துளை வழி மேல்தளத்திலிருந்து இ இறங்கிவரும் மின்சார ஏணி, குடைவுச்சுவர் வழி ஒழுகும் நீர், மேற்கூரை இற்றுவிழும் ஓட்டை ஏணி, ஏணியின் துரு வாடை, கிரீ சின் எண்ணெய் கமறல் ஏற்படுத்தும் குமட்டல் வாடை , இருளில் ஏணி செல்லும்போது கூரையிலும் சுவர்களிலும் 
புரண்டோடும் ஊற்றுவெள்ளம், சுவர்ப்பக்கவாட்டு வாய்க்காலில் மழைவெள்ள இரைச்சல், ராட்சத மின் எந்திரங்கள் பள்ளங்களில் சேகரமான நீரை உறிஞ்சி தாரையாகக்கொட்டுவது ஏணியிலிருந்து இறங்கிய தொழிலாளர்கள் ஒரேசமயத்தில் தங்களது ஹெல்மெட்கள் மூலம் இயக்கும் பேட்டரி வெளிச்சம் குறுக்களவு பதிமூன்று அடி - உயரம் ஒன்பது அடியுள்ள குடைவுப்பாதைகள், ஐம்பது அடி தடிமனான கிளைக் குடைவுத் தூண்கள் கரிப்படிவம் வரையிலான தோண்டுமிட எல்லைகள் என்ற சித்திரிப்புகள் யதார்த்தமான சுரங்கப்பாதைக்குள் திகிலுடன் நம்மைப் பயணிக்க வைப்பன.

\section{கதை மாந்தர்கள்}

\section{பிகாஸ் சௌதரி}

கதை எழுதத் தூண்டிய பிகாஸ் சௌதரியின் சொந்த தொழிற்சங்க வாழ்க்கை அனுபவங்கள் குசிபா. விடம் பகிரப்பட்டதால் இதன் நாயகன் பெயரும் பிகாஸ் சௌதரியாகவே அமைந்துவிட்டது. அரசியல் தொழிற்சங்க வாழ்க்கைக்குத் தம்மை அர்ப்பணித்துக் கொண்டதால் நிஜ மனிதர் தோழர் பிகாஸ் சௌதரி இறுதி வரை திருமணம் செய்து கொள்ளவில்லை. (நாடாளுமன்ற உறுப்பினர் பிகாஸ் சௌதரி குறித்த நாடாளுமன்ற இணையதள விவரக்குறிப்பு இதை உறுதிப்படுத்துகிறது. மேற்கு வங்க மாநில சிபிஐ - எம் கட்சியின் மாநிலக்குழு உறுப்பினராகவும், மேற்கு வங்க மாநில இந்திய தொழிற்சங்க மையத்தின் (சிஐடியு) மாநில செயற்குழு உறுப்பினராகவும், நான்கு முறை மேற்கு வங்க சட்டப்பேரவை உறுப்பினராகவும் இருமுறை நாடாளுமன்ற அவை உறுப்பினராகவும், மத்திய அரசின் நிலக்கரித்துறை தொடர்பான ஆலோசனைக் குழுக்களிலும் உறுப்பினராக இடம்பெற்றவர்.) புதின நாயகனும் இவரைப்போலவே தொழிற்சங்கவாதி மார்க்சிஸ்ட் கட்சி உறுப்பினர். 'தத்துவப் பார்வையும் அரசியல் வழிகாட்டுதலும் தீர்மானம் மேற்கொள்வதில் தெளிந்த ஞானமும் பெறவேண்டும். அத்தோடு நிறையப் படிக்க வேண்டும் என எண்ணினான். அன்றாட உழைக்கும் மக்கள் நடத்தும் கோரிக்கைகளுக்கான போராட்டத்தையும் அரசியல் பொருளாதார நடவடிக்கைகளையும் கற்றுத்தெளிவடைய 'கணசக்தி' பத்திரிகை யைத் தற்பொழுது முறையாகப் படிக்க ஆரம்பித்தான். "கணசக்தி" என்பது மேற்கு வங்க மாநிலத்தின் சிபிஐ (எம்) கட்சி வெளியிட்டுவரும் வங்காள நாளிதழ் ஆகும்.

\section{ஜமுனா குமாரி}

பிகாஸ் பெற்றோர் இருவரும் காலமான பின்னர், தாய் ஸ்தானத்திலிருந்து வளர்த்துப் படிக்க வைத்த பிகாஸின் அக்கா. தம்பிக்குத் திருமணம் செய்து குடியும் குடித்தனமுமானால் வாழ்க்கை முன்னேறிவிடும் என்று நம்புபவள். ஒவ்வொரு முறையும் சுரங்கத்தில் தொழிற்சங்கத்தின் வேலை நிறுத்தம், மறியல் நடக்கும் போதும் நிர்வாகம், காவல்துறையின் தாக்குதலுக்கு ஆளாகும் பிகாஸ், ' துர்காதேவி அருளால் உயிருக்கு ஆபத்தில்லாமல் தப்பிப்பதாக' நம்பும் ஜமுனா குமாரி தம்பியின் சமூக வாழ்க்கைக்குத் தடை செய்யவில்லை. எனவே தம்பியின் தொழிற்சங்க சமூக வாழ்வு இடையறாது தொடர்கிறது.

\section{சகுந்தலா தேவி}

வஞ்சகனான சுரங்க முதலாளி திவாரியின் மனைவி சகுந்தலா தேவி கருணைமிக்க ' சூழல் கைதி.' பூர்வீக கிராம வாழ்க்கையை ஏக்கத்துடன் அசைபோடுபவள். ஏழையான தன் பெற்றோருடன் உறவு அறுபடக் காரணம் கணவனின் ' திடீர் ' பணக்கார புத்தியும், பணக்கார ஒழுக்கக்கேடும் என்பதை நன்குணர்ந்தவள். அமைச்சர் பெருமக்கள் உள்ளிட்ட 'சமூக உயர்வட்டாரம்' பங்கேற்கும் தமது மகளின் ஆடம்பரத் திருமண நிச்சயதார்த்தத்தில் தன் பெற்றோர் பங்கேற்பது இழிவு எனக் கணவன் கருதவே மனவேதனை கொள்கிறாள் அவனைப்பழிக்கிறாள். அவ்வப்போது பல பெண்களுடன் பாலியல் சகவாசம் வைத்திருக்கும் தன் கணவனை அறிவாள் தட்டிக்கேட்டு தனது மணவாழ்வு முறிந்துபட்டால் வரும் வலியை உணரக்கூடியவள் என்பதால், எதிர்க்க இயலாது அமைதி காக்கிறாள். ஆனால் தன்னையொத்த 
சீமாட்டிகளைப் போல, கணவனின் ஒழுக்கக்கேட்டிற்குத் துணை போகும் பாத்திரமல்ல. பொதுவாக வில்லன் ' என்றாலே அவன் மனைவியும் அப்படித்தான் என்று சித்திரிக்கும் இலக்கிய, திரைப் பாத்திரங்களைப் போலல்லாதது சகுந்தலாதேவியின் பாத்திரப்படைப்பு.

\section{குடிகாரத் தொழிலாளர்கள்}

உழைப்புச்சுரண்டலும், கந்துவட்டிக் கொடுமையும் சேர்ந்து பல தொழிலாளர்களை சாராயக்கடைக்கு அனுப்புகிறது. உடலுழைப்பு அலுப்பில் நிம்மதி கிடைப்பதான உணர்வு எழுவதால் சுரங்கத்தைவிடவும், வசிக்கும் எலிவளைக்குடிசைகளை விடவும் சாராயக்கடை இவர்களுக்குப் பிடித்தமான இடமாக அமைகிறது.

தங்கள் உடல் அலுப்பையும் மனச்சோர்வையும் சற்று நேரம் மறந்திருப்பதற்கு கிடைத்திருக்கும் பூலோக சொர்க்கம் அது. முதலாளிகளில் இருந்து தொழிலாளர்கள் வரையிலான வாழ்க்கை சம்பந்தப்பட்ட எல்லா விசயங்களும் ஒளிவுரகசியமின்றிப் பேசப்படும் இடம். (ப. 106) சூபர்வைசர், மைனிங் சர்தார் குறித்து ஒருவன் பேசியதை, "இவனுகளுக்கெல்லாம் மானமே கெடையாது. தங்கள் பொண்டாட்டி தங்கச்சிகளே வுட்டுக் குடுத்து வயிறு பொழைக்கற தேவடியா நாய்க, நம்ம வயித்திலே அடிச்சி தங்கள் வயித்தே ரொப்பிக்கிறானுக", என்று போதையில் உளறியதை மைனிங் சர்தாரே தன் காதுபடக்கேட்டு அதனால் மாதச்சம்பளத்தை இழந்து ஈட்டிக்காரனிடம் 'நாயே' என்று உதை வாங்குபவனும் உண்டு. 'நந்தங்கதை தெரியுமா?' என்று ஒருவன் சகதொழிலாளி குறித்துப் பேச ஆரம்பிக்க,'காந்தியின் அவதாரமாச்சே அவன்' 'நம்மளப் பார்த்து குடிகாரப்பசங்களே! குடிக்கிற பணத்தை மிச்சப்படுத்தி மாடுவாங்கி பாலூத்திப் பணம் சேத்து நெலம் வாங்கி கசுமால வேலய வுட்டுப்போறேன்னு சொன்வனாச்சே!' என்று இன்னொருவன் முனக, முதலாமவன் 'அவன் மாடு நேத்துச் செத்துப்போச்சு' என்று சொல்ல' நல்லகாலம். நாமநேத்து குடிக்க வராமப்போனோம். கடவுள் காப்பாத்திட்டாரு' என்று சொல்கிறான்!

'மசுருக்கடவுளு நாம கொளந்தை குட்டிகளோட பட்டினி கெடந்து சாவறோம். அப்ப இந்தக் கடவுளு தூங்கிட்டானா? மொள்ளமாறிச் சாமியேத் தூக்கி கொளத்துலே போடணும்டா" என்று மூன்றாமவன் கூறுகிறான். 'கரிவண்டி கொட்டற அப்பன் பஸ்வான் சிபாரிசுல வேலைக்குவந்த, புருசனோட வாழாமே வந்துட்ட சாவித்திரிக்கு வேலை செய்யாமக் கூலி கெடைக்கும்' அரசல்புரசலான 'ரகசியம்' பரிமாறப்படுகிறது. 'சிரிச்சு மயக்கி மேனேஜரை அவள் மடியில கிடத்தி அவள் வேலையை மத்தவங்களச் செய்ய' வைக்கிறாளாம். மொதலாளி பங்களா காவல்காரன் நோயில் படுக்க அவன் காவல் வேலையைப்பார்க்கப் போன ஒருவன், 'மொதலாளி ஒரு பொம்பளையோடு படுத்துக் கெடக்கும்' நிலையைப் பார்க்க நேர்ந்த கதையைக் கூறுகிறான். 'இன்னிக்கு என்ன கொண்டாந்தீங்கன்னு அவ கேட்க, தங்கச்சங்கிலி கோத்த தங்க அரமூடிய அவ இடுப்புல கட்டித்தொங்கவிட்டு அவ மானத்த மூடுன' கதையைச் சொல்லிப் புலம்புகிறான். 'நம்ம பொம்பளைங்களுக்கு மாத்துடைக்கு கந்தல் துணி கூடக் கெடைக்க மாட்டேங்குது. மொதலாளி நம்ம சதையைப் பிழிஞ்சு காசு சேர்த்து கூத்தியாளுக்குத் தங்க அரமூடி பண்ணிப்போடறான்' என்று வரும் குறிப்புகளிலிருந்து சாராயக்கடைகள் வம்பளக்கும் இன்பக்கேணிகளாகத் தோன்றுகின்றன. உளறல் என்று எளிதில் ஒதுக்கமுடியாதபடி, அவ்வப்போது நக்கல் செய்யும் பாத்திரங்களான இவர்களுக்கு நிகரில்லை. நாடகம், திரைப்படங்களின் போக்கில் அவ்வப்போது ஏற்படும் சலிப்பைப் போக்கும் நகைச்சுவைக் காட்சி இடையீடு (Comic relief) போன்று இவர்கள் பங்கேற்கும் காட்சிகள் அமைகின்றன. சமூக அழுக்குகள் அலசப்படும் அங்கதமாக இவை அமைகின்றன. ஆங்கில இலக்கிய நாடகாசிரியர் வில்லியம் ேேக்ஸ்பியரின் ' முட்டாள்கள் '(Fools) பாத்திரங்கள் தற்கொலையான ஒபீலியா உடலை கிறிஸ்தவ முறையில் அடக்கம் செய்யும் வெட்டியான்கள் (Grave-diggers) தங்களுக்குள் பாடி உரையாடும் போது உதிர்க்கும் தத்துவம் (“ஹேம்லட்" துன்பியல் நாடகம்) சித்தர்களின் சிந்தனை கிளறும் உடல் அழிவுத் தத்துவம் கானாப்பாடல்களில் ஒலிக்கும் ஆவேசம் ஆகியவற்றை இங்கு ஒப்பிடலாம். கந்துவட்டிக்காரர்களிடமிருந்து தப்பித்து கூலியைக் காக்க வழிதெரியாத தொழிலாளர்கள், ஒவ்வொரு மாதமும் பொருத்தமான பொய்யைச் சொல்கிறார்கள் உள்ளாடை, மலக்குடலுக்குள் பணத்தை ஒளித்துவைத்துத் தப்பிக்கும் ஒருவனுமுண்டு. 


\section{மனித மாண்பிழந்த முதலாளித்துவம்}

இங்குள்ள தொழிலாளர்கள் சாராயக்கடைகளில் மட்டும் பாக்கி வைப்பது, பெரும்பாலும் இல்லை. பலவிதங்களில் சுரங்கத்தொழிலாளியின் கூலியின் பெரும்பகுதி சாராயக்கடைகளுக்குச் செல்வதை சுரங்க முதலாளிகள் நன்கு அறிவார்கள். முதலாளி வர்க்கமும் அதன் அடிமைகளும், தொழிலாளர்கள் இப்படி அரைகுறை உணர்வுடன் இருப்பதையே விரும்புகின்றன. இவர்கள் சிந்திக்கத் தொடங்கிவிட்டால் கொடிப்பிடித்து தொழிற்சங்கம் வைத்து தமது லாபத்திற்கு வேட்டுவைத்து விடுவார்கள் என்ற அச்சமே இதற்குக் காரணம்.

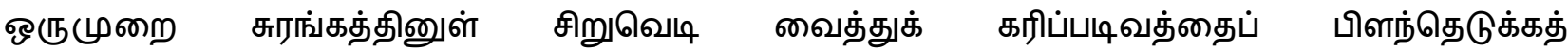
துளையிடும்பொழுது தண்ணீர் பீய்ச்சியடிக்கத் துவங்கி விட்டது. அதை உடனடியாக அடைத்து விடும் முயற்சியில் ஈடுபடாவிட்டால் பெரிய விரிசல் ஏற்பட்டு நீர்ப்பிரவாகம் எடுத்து விடும். உள்ளே உள்ள அத்தனை பர்களுக்கும் ஆபத்து ஏற்படும். இந்நிலையில் ஒரு தொழிலாளி தன் முதுகை அத்துவாரத்தின் மீது சாய்த்து தன் பலங்கொண்ட மட்டும் அமுக்கி நின்று கொண்டான். தொழிலாளர்கள் முட்டுப்பலகையை எடுத்தோடி வந்தனர். உடன் புதிய நெருக்கடி ஒன்று உருவாகிவிட்டது. அவன் அமுக்கிக் கொண்டிருந்த துவார வடும்பை ஒட்டி தண்ணீர் பீய்ச்சியடிக்கத் துவங்கிவிட்டது. அவன் நகர்ந்து ஒதுங்கினால் போதும், சுவரில் பெரும்பிளவு உருவாகிவிடும். இதையுணர்ந்த பொறியாளர் உடன் ஆபத்தைத் தடுக்க அமுக்கிக் கொண்டிருந்த தொழிலாளி மீதே பலகைகளை அழுத்தி முட்டடிக்க உத்தரவிட்டான். சிலுவையில் அறையப்பட்ட ஏசுவைப் போல உயிரோடு அத்தொழிலாளி சுவற்றில் அறையப்பட்டான். தன் சக தொழிலாளியின் உடல் மீது மனதைக் கல்லாக்கிக் கொண்டு மற்றவர்கள் அவ்வேலையைச் செய்து முடித்தனர். அவர்களால் மறுத்துப் பேசமுடியாத நிலை. முதுகை அத்தொழிலாளி நகர்த்தி விட்டால் அங்குள்ள தொழிலாளர்கள் அனைவரும் உடைப்பெடுக்கும் அணைவெள்ளம் போலப் பீறிட்டெழும் சுரங்க உடைப்பு நீரில் மூழ்கவேண்டி நேர்ந்துவிடும். சுரங்கப்பாதுகாப்புக்காகவும் தன் சக தொழிலாளர்களின் உயிருக்காகவும் அவன் தவிர்க்க முடியாமல் தன் உயிரைத் தியாகம் செய்தான். இதோடு இல்லை சுரங்க முதலாளி அத்தொழிலாளியின் உயிர்த் தியாகத்தைக் கண்டுகொள்ளவேயில்லை. மற்றத் தொழிலாளர்களின் உயிரைக் காக்க அவன் தன் உயிரை மாய்த்துக்கொண்டான். இதில் நான் என்ன செய்ய முடியும்? அவன் குடும்பப் பாதுகாப்பிற்கு நான் எதற்கு உதவவேண்டும்? நான் கூறி அவன் தன்னை சுவற்றில் அறைந்து கொள்ளவில்லையே? சுரங்கம் நீர் பீறிட்டு மூழ்கி விட்டால் காப்பீட்டுத் துறையிடம் நட்ட ஈடு வாங்கிக் கொள்கிறேன். யானை இறந்தாலும் ஆயிரம் பொன் இருந்தாலும் ஆயிரம் பொன் என்பார்கள். அப்படித்தான் சுரங்கம். அதுவுமில்லாமல் சுரங்கமும் கிட்டத்தட்ட கரி இருப்புக் குறைந்து விட்டது. அழிந்தால் காப்பீட்டுத் தொகையைக்கொண்டு புதுச்சுரங்கம் வாங்கிக் கொள்வேன்..." என்னும் பகுதி வாசகர்களைச் சில்லிடவைக்கிறது! முதலாளித்துவ சுரண்டலின் குரூர வேட்கை இதுதான்! உழைப்பு தொழிலாளரை அந்நியப்படுத்துகிறது. அந்நியமாதல் (Alienation) என்பது ' பிறருக்குச் சொந்தமானதைப் பலவந்தமாகப் பறிப்பதும், ஓர் உடைமையைக் கைப்பற்றித் திசைதிருப்புவதுமே ஆகும்.' என்னும் மார்க்சியச் சொல்லகராதி கூற்று இங்கு கவனிக்கத்தக்கது. (Rius, 2017)

\section{மார்க்சிய நாத்திகத்தின் மனிதநேயக்கூறு}

மூடநம்பிக்கை எதிர்ப்பு, அறிவியல் கண்ணோட்டம் என்பன பகுத்தறிவு நோக்கில் மார்க்சியக்கூற்றின் ஒரு பகுதி. இதர பகுத்தறிவுவாதிகளிடமிருந்து மார்க்சியர்கள் வேறுபடுமிடமும் இதுதான். கிண்டல் பண்ணுவது மட்டுமே பகுத்தறிவு ஆகிவிடாது. நம்பிக்கை உள்ளவர்களின் சூழலிருந்து பார்த்துப் பொறுமையோடு காலங்கனிந்த சூழலில் இதை நாசூக்காக வெளிப்படுத்தி விவாதிக்கவேண்டும். அறிவுப்புலத்தில் காரசாரமான விவாதங்கள் மேற்கொள்ள முடியும். ஆனால் படிப்பறிவற்ற, பகுத்தறிவுச் சிந்தனையற்ற எளிய அடித்தட்டு மக்களை அறிவியல் கண்ணோட்டத்துடன் வென்றெடுக்க, அசாத்தியப் பொறுமை தேவை. மார்க்சிஸ்ட் கம்யூனிஸ்ட் கட்சியின் தலைமையிலான கேரள இடதுசாரி ஜனநாயக அரசு நிதானமாக, மங்களுக்குத் தொடர்புடைய, மக்கள் நல்லிணக்கம் பேணும் ஆத்திக விஷயங்களை அலசி, ஜனநாயகத்தன்மையோடு உரிய அமைப்புகளில் பேசி, 
முடிவெடுக்கிறது. பெண்களுக்கு சமவாய்ப்பு வழங்கும் வழிபாட்டு உரிமையைக் கோரி நிற்கிறது. ஓணம் பண்டிகையை எல்லா சமயநம்பிக்கை கொண்டவர்களும் கொண்டாடுவது கேரளத்தின் சிறப்பு. அரசியலிலிருந்தும், கல்விக்கூடங்களிலிருந்தும் மதத்தைப் பிரித்தெடுத்து வகுப்பு நல்லிணக்கத்தை அமுலாக்குவதுதான் மார்க்சிய இலக்கியவாதி ஈ. எம். எஸ். நம்பூதிரிபாடு தலைமையிலான கேரள இடது ஜனநாயக முன்னணி அரசின் சாதனை அந்த மரபு முதல்வர் பிணராயி விஜயன் வரை இன்றளவும் தொடர்கிறது. வகுப்பு நல்லிணக்கம் தொடர்பான எண்ணற்ற நூல்களை எழுதியுள்ளவரும், காலடியிலுள்ள சங்கரர் சமஸ்கிருதப் பல்கலைக்கழகத்தின் மேனாள் துணைவேந்தரும், மார்க்சிய அறிஞருமான பேராசிரியர் கே. என். பணிக்கர் ஓணம் பண்டிகையை அனைவரும் கொண்டாடும் சமயச்சார்பின்மைத் திருவிழாவாகக் காண்கிறார். மேற்கு வங்க மாநில துர்கா பூஜைக் குழுக்களில் சமய நம்பிக்கையற்ற மார்க்சியர்களையே பல இடங்களில் ஒருங்கிணைப்பாளர்களாக அங்குள்ள தலக்குழு மக்கள் தேர்வு செய்கின்றனர்! (இதை, இக்கட்டுரையாளர் மேற்கு வங்க ஜனநாயக எழுத்தாளர் சங்கத்தின் மேனாள் பொதுச்செயலாளர் இந்திரநாத் பாந்தோபாத்யாய- 'பானர்ஜி' - அவர்களுடனான "தீக்கதிர்" நாளிதழ் நேர்காணலின்போது உரையாடி அறிந்து எழுதநேர்ந்தது. தோழர் இந்திரநாத் தமது நண்பர் என்று குசிபா (Chinnappa Bharathi, 2012).

மதவெறிக்கு இரையாகிவிடாமல் மார்க்சியர்கள் வழிநடத்திச் சென்ற அனுபவம் காரணமாக வகுப்பு நல்லிணக்கம் ோதிபாசு முதல்வரான காலத்திலிருந்தே இடதுமுன்னணி ஆட்சியிலில்லாத போதும் - மரபாகத் தொடர்கிறது. எனவேதான், தனது அக்கா இறைநம்பிக்கை கொண்டவளாக இருந்தாலும் அவளது நம்பிக்கைக்கு எதிரான தமது கருத்துக்களை வெளிக்கூறாது அடக்கிக் கொண்டு, அவளது பார்வையிலிருந்து நோக்கி, புரிந்து கொண்டு செயல்படுகிறான் ' சுரங்கம் ' நாயகன் பிகாஸ் சௌதரி. திருமணம் தற்போது தேவையில்லை என்பதை சூக்குமமாக வெளிப்படுத்துகிறான்.

\section{பண்பாட்டு வழக்கங்கள், சடங்குகள்}

வங்கக் கலாச்சாரத்தின் சில கூறுகள் சுரங்கமுதலாளி திவாரியின் மனைவி சகுந்தலா தேவியின் மூலமாக வெளிப்படுத்தப்படுகின்றன. பெருவிரல் நகங்களுக்கிடையில் பேனை நசுக்கிவிட்டு 'ஸ் ' கூறுவது வங்கக்கலாச்சாரம். அப்படிக் கூறாவிட்டால் பேனுக்கு சொர்க்கம் கிட்டாதாம். கூடவே சகுந்தலாதேவி சிரித்துக்கொண்டே சொல்வது, 'மனுசன் பாவத்தைக் கழுவவே பறவையாய் பறந்து திரியறப்போ பேனுக்கு சொர்க்கம் கேக்குறதுதா கொறையா இருக்குது போல...' என்று ஏகடியம் பேசுகிறாள் சகுந்தலா தேவி. தமிழகத்திலும் நெல்லைப்பகுதியில் பேன்களை நகங்களுக்கிடையே நசுக்கும் மூதாட்டிகள் 'ஸ்' என்றே தமக்குள் ஒலிக்கின்றனர்! மாலையில் பசுக்கள் மேய்ச்சல் நிலத்திலிருந்து திரும்பும்போது தெருவில் அவற்றின் கால் மிதிபட்டு தூசி பரவும் நேரமான 'கோதூல ' நேரத்துக்குப் பிறகே திருமணத்துக்கு நல்ல லக்னம் அமையுமென்பது வங்காளிகளின் நம்பிக்கை.

\section{வேதமரபு மணச் சடங்கு வழக்கங்கள்}

புரோகிதர் சமஸ்கிருத மந்திரம் சொல்லி ஹோமம் வளர்த்துப் பூஜை செய்யும்போது மணப்பெண்ணை சிவப்பு நிறப்புடவையான 'சேலி' அலங்கரிக்க ' கோம்டா ' (முக்காடு) இட்டபடி தோழிகளின் கேலி, விஷமத்தூண்டலுடன் அமரவைத்துப் பலகையோடு தூக்கி ஹோமத்தீயை 'சர்பாத்' (ஏழுமுறை சுற்றுதல்) செய்தல், சுபதிருஷ்டி, மணமகளின் உச்சி வகிட்டில் சிந்தூரம் தடவி இரும்பு வளையம், 'சங்கா' (சங்கு வளையல்) அணிதல், 'உலு உலு' குலவையொலி, 'சப்தபதி' (ஏழு அடிகள் நடத்தல்), 'லாஜ்' ஹாமத்தில் மணமகள் ோள்ளுக்கப்பால் நெல்பொரி போடும் சடங்கு உண்ணாவிரதச் சடங்கில் இருக்கும் மணமக்களை தனிஅறைக்குள் இணையவிடாது தோழிகள் 'தொந்தரவு செய்து' கூச்சம் போக்கும் 'வாசர் கர்' சடங்கு 'பாஸிபியே' (பழசான திருமணம்) 'ஸ்த்ரீ ஆசார்' (மணப்பெண்ணுக்கு அன்னியோன்யம்- நிகழ்த்து சடங்கு) 'கால் ராத்ரி' (கெட்ட இரவு, இணையர் பிரிந்த இரவு) மணமகள் சகோதரியுடன் மட்டுமே இருப்பது 'பௌபாத்' (மணமகன் உறவினர்களுக்கு மணமகள் பரிமாறும் சடங்கு) பண்பாட்டு மானுடவியல் கூறுகளாக இவை காணப்படுகின்றன. 


\section{மார்க்சியக் கோட்பாடுகள்}

மார்க்சியத் தத்துவம், மார்க்சியத்தின் இயக்கவியல் கூறுகளான இயக்கவியல் பொருள்முதல்வாதம், வரலாற்றுப் பொருள்முதல்வாதம், மார்க்சியம் குறித்த வரையறை, மார்க்சிய இலக்கியக் கோட்பாடுகள், அடித்தளம் - மேற்கட்டுமானம், பிரதிபலிப்புக் கொள்கை, யதார்த்தவாதம், சோஷலிச யதார்த்தவாதம், வரலாற்றுச்சூழமைவு உள்ளிட் மார்க்சியக் கலைச்சொற்களின் விளக்கங்களை அறிந்தபின்னர், 'சுரங்கம்' புதினத்திற்கு மார்க்சிய இலக்கியப் பொருத்தப்பாட்டை நாம் உணரமுடியும்.

\section{மார்க்சியத்தின் இயக்கவியல் கூறுகள்}

அளவில் ஏற்படும் மாற்றம், குணத்தில் மாற்றத்தை ஏற்படுத்தும். அதேபோலக் குணத்தில் ஏற்படும் மாற்றம் அளவில் மாற்றத்தை ஏற்படுத்தும். எதிர்மறைகளாக இருந்தாலும் ஒன்று மற்றொன்றில் ஊடுருவும். ஒருநிலை இருந்தால் அதற்கு எதிர்நிலை இருக்கும். வளர்ச்சிப்போக்கின் ஒரு கட்டத்தில் இந்த இரண்டு நிலைகளும் ஓன்றிக் கலந்து புதிய நிலையாக மாறும்.

\section{வரலாற்றியல் பொருள் முதல் வாதம்}

சமூக வாழ்நிலையே, இருத்தலே மனிதரின் உணர்வைத் தீர்மானிக்கிறது. மனிதரின் உறவு சமூக வாழ்நிலையைத் தீர்மானிப்பதில்லை. உற்பத்தி முறையே மனிதரின் உணர்வைத் தீர்மானிக்கிறது. அரசு, கருத்தியல், மதம் ஆகியன உற்பத்தி முறையால் தீர்மானிக்கப்படுகின்றன. குறிப்பிட்ட சமுதாய வளர்ச்சிக் கட்டத்தில் உற்பத்தி உறவுகள், உற்பத்தி சக்திகளுடன் மோதுகின்றன. உற்பத்தி சக்திகளின் வளர்ச்சிக்கு உற்பத்தி உறவுகள் தளை ஆகின்றன. இதன் விளைவாக சமுதாயப் புரட்சி உற்பத்தி உறவுகளை மாற்றுகிறது. அதனோடு சேர்ந்து அரசு, பண்பாடு முதலியனவும் மாறுகின்றன என்று மார்க்சியம், தமிழ்ச் சிற்றிதழ்களின் வழி உருவான நவீனத் திறனாய்வுப் போக்குகள் நூலின் மார்க்சியம் என்ற அத்தியாயத்தில் மேற்கண்டவாறு குறிப்பிடுகிறார் (Kamarasu, 2016a).

\section{மார்க்சியம்- வரையறை என்ன?}

"மார்க்சியம் என்பது கார்ல் மார்க்சினுடைய கருத்துக்களின் அமைப்பு ஆகும். கருத்துக்களின் தொகுப்பு என்பது வேறு. அமைப்பு என்பது வேறு. மார்க்ஸ் பல்வேறு துறைகளில் ஆராய்ச்சி செய்து புதிதாய்ப் பலவும் கண்டுபிடித்து, தாம் அடைந்த முடிவுகளைக் கோட்பாடுகளாக வகுத்துரைத்தார். இந்தக் கோட்பாடுகளுக்கிடையே ஒரு புதிய உலகக்கண்ணோட்டத்தின் முரணற்ற மைய இழையைக் காணமுடிகிறது. அவருடைய கருத்துகளிடையே உயிரோட்டமான தொடர்பு உள்ளது. அவை திண்ணியமானதொரு உடலமைப்பின் வெவ்வேறு உறுப்புகளைப்போல் இயைந்து செயல்படுகின்றன. அதனால்தான் மார்க்சின் கருத்துகள் ஒரு கதம்பத் தொகுப்பு போலல்லாமல் உயிர்ப்பான ஒழுங்கமைப்பாய் உருப்பெற்று மார்க்சியம் என்னும் அறிவியல் கருத்தமைப்பாக ஏற்றம் பெறுகின்றன என்று "மார்க்சியம்: அனா, ஆவன்னா" என்று தாம் எழுதிய நூலில் வரையறை தருகிறார் (Thiyagu, 2019). "மூலதனம்" (மூலமுதல்) நூலை சிறையிலிருந்த காலத்தில் தமிழாக்கம் செய்ததை, சிறையிலிருந்து வெளியானபின் ஏழாண்டு உழைப்பில், ரா. கிருஷ்ணையா சீரமைப்பில், ' என்சிபிஎச் ' பதிப்பகத்திற்காகத் தமிழில் தந்தவர் (Thiyagu, 2019).

\section{மார்க்சிய இலக்கியக் கோட்பாடுகள்}

"மார்க்சியம் அடிப்படையில் ஓர் அரசியல் பொருளாதார சமுதாயத் தத்துவம். இதன் மூலவர்களும் முன்னவர்களுமான மார்க்சும், எங்கெல்சும் பின்னவரான லெனினும் இலக்கியக் கோட்பாடுகள் பற்றித் தனியாகவோ முழுமையாகவோ கூறவில்லை. அவர்கள் பொருளாதாரக் கோட்பாடுகள், அரசியல் கொள்கைகள், சமுதாயப் போக்குகள் குறித்து விரிவாக விவாதித்துள்ளனர். 
அதே நேரத்தில் கலை இலக்கியத்தில் ஆழ்ந்த ஈடுபாடும் ஞானமும் மிக்கவர்களாகவும் பண்டைக்காலம் முதல் சமகாலம் வரையான கலை இலக்கியக் கருவூலங்களைக் கையாளக்கூடியவர்களாகவும் திகழ்ந்தார்கள். பல படைப்பாளிகளுடன் நெருங்கிய நேரடித் தொடர்புகளும் கொண்டிருந்தனர். கலை இலக்கியங்கள் குறித்தும் படைப்பாளிகள் பற்றியும் பல இடங்களில் அவர்கள் விவாதித்துள்ளனர். இதன் மூலம் கலை இலக்கியம் பற்றிய பண்புகளைச் சுட்டியுள்ளனர். இவையும் பின்னர் மார்க்சியத்தை ஏற்றுக்கொண்டு வளர்த்தெடுத்த பல மார்க்சியர்களின் கருத்துகளும் இணைந்து மார்க்சிய அழகியலை மார்க்சிய இலக்கியக் கொள்கையை உருவாக்கியுள்ளன." என்கிறார் (Kamarasu, 2016a).

\section{அடித்தளம் மேற்கட்டுமானம், பிரதிபலிப்புக் கொள்கை}

"Base or (infra) and Superstructure அடித்தளம், மேற்கட்டுமானம் / உற்பத்தி முறை, அதாவது உற்பத்தி சக்திகளும் உற்பத்தி உறவுகளும், பொருளாதார அடித்தளத்தை அமைக்கின்றன. (அதாவது, சமுதாயத்தின் அடித்தளத்தை). அடித்தளம் (அல்லது உள்கட்டுமானம்) மாற்றியமைக்கப்பட்டதும், பின்னர் மேல்கட்டுமானமும் (அரசியல் அமைப்பு முறை, மதம், தத்துவம், ஒழுக்க நெறி, கலை, அறிவியல் இ இன்னபிற.) தன்னை மாற்றிக்கொள்ளும். ஏறத்தாழ விரைவான வகையில்" (Rius, 2017). என்கிறது ரியூஸ் மார்க்சியச் சொல்லகராதி. "மார்க்சியத் தத்துவத்தின் அறிவுத்தோற்றவியல் அடிப்படையில் லெனினால் வளர்த்தெடுக்கப்பட்ட இலக்கியக் கோட்பாடு (Theory of Reflection)," என்கிறார் (Kamarasu, 2016a).

\section{யதார்த்தவாதம்}

"தமிழில் யதார்த்தவாதம் நவீனத்துவத்துக்கு மறுதலையாகவே எழுந்தது. பெரும்பாலும் மார்க்சியம் சார்ந்தவர்களே இதற்கு அழுத்தம் கொடுத்தனர். நவீனத்துவ எழுத்து அலைக்கு எதிராக எழுந்த சாந்தி, சரஸ்வதி, மனிதன், சமரன், தாமரை, செம்மலர், சிகரம், வானம்பாடி முதலிய இதழ்களில் யதார்த்தவாதப் படைப்புகளும் திறனாய்வுகளும் இடம்பெற்றன. தொ.மு.சி. ரகுநாதன், க. கைலாசபதி, கா. சிவத்தம்பி, நா. வானமாமலை, எஸ். தோதாத்ரி, பொன்னீலன், கே. முத்தையா, தி.சு. நடராசன், அருணன் போன்றோர் யதார்த்தவாதம் குறித்து எழுதி உள்ளனர் என்கிறார். (Kamarasu, 2016b).

\section{சோஷலிச யதார்த்தவாதம்}

"யதார்த்தம் (Realism) என்பது படைப்பில் உள்ளடக்கமும் உத்தியும் இணைந்து பரிணமிக்கும் ஒன்றாகும். யதார்த்தத்தை ஒரு சக்தி வாய்ந்த கருவியாக மார்க்சிய மூலவர்கள் கருதினர். இதனை எவ்வாறு இலக்கியத்தில் உருவாக்கவேண்டும் அல்லது எப்படி இலக்கியத்தில் அமைந்திருக்கிறது என்பதைப் பல இடங்களில் சுட்டுவர். யதார்த்தவாதம் (Realism), சமூக யதார்த்தவாதம் (Social Realism), விமர்சன யதார்த்தவாதம (Critical Realism), இயற்பண்புவாதம் (Naturalism) ஆகியவற்றின் வளர்ந்த நிலையாக சோஷலிச யதார்த்தவாதம் (Socialist Realism) விளங்குகிறது.

எஸ். தேதாத்ரி "சோஷலிச யதார்த்தவாதத்தின் அடிப்படைகள்" எனும் தமது நூலில் இதன் பண்புகளைக் கீழ்க்காணுமாறு கூறுவார். "உலக இலக்கிய அரங்கில் யதார்த்தவாதம் வளர்த்து வைத்திருந்த விதிமுறைகளை அடியொற்றியே இது (சோஷலிச யதார்த்தவாதம்) எழுந்தது. யதார்த்தவாதத்தில் காணப்படும் சமூக ஆய்வு முறை, வகைப்பாடான பாத்திரப்படைப்பு, சமூகத்தைப் புறவயமாக (objective) ஏற்றுக்கொள்ளுதல் ஆகியவை இங்கு தொடருகின்றன. சமூக விமரிசனமும் ஆழமாக இடம் பெறுகிறது. இவற்றிற்கெல்லாம் மேலாக சோஷலிச யதார்த்தவாதத்தில் வேறு சில புதிய அம்சங்களும் இடம்பெறுகின்றன. உழைப்பு மனிதனது வாழ்வின் அடிப்படை என்ற நோக்கம் இந்த இலக்கியங்களில் காணப்படுகிறது. சமூகம் இயங்கியல் பொருள்முதல்வாத முறையில் ஆய்வு செய்யப்படுகிறது. வர்க்கப் போராட்டத்தின் தன்மை சித்தரிக்கப்படுகிறது. உழைப்பவனைச் சார்ந்து நிற்கும் போக்கு காணப்படுகிறது. இதனையொட்டிய வரலாற்று உணர்வும் மனிதநேயமும் சமூக 
உணர்வும் மற்ற இலக்கியங்களில் காணமுடியாத அளவிற்கு இந்தவகை இலக்கியங்களில் மிகவும் விரிவாக இடம்பெற்றிருப்பதைக் காணமுடிகிறது." (Thiyagu, 2019).

அடித்தளம்- மேற்கட்டுமானம், பிரதிபலிப்புக் கொள்கை, சோஷலிச யதார்த்தவாதம் எனும் இவ்வடிப்படைகளோடு சேர்த்துக் கவனத்தில் இடம் பெற வேண்டுவது உருவம் - உள்ளடக்கம் பற்றிய சிந்தனையும் ஆகும். உள்ளடக்கம் முதன்மையானது. அதே வேளை உருவம் ஒதுக்கப்படக் கூடியதும் அல்ல. அது உள்ளடக்கத்தைப் பாதிக்கும் வளர்ச்சிக்கு இட்டுச்செல்லும் என இரண்டின் முக்கியத்துவத்தையும் மார்க்சியம் விளக்குகிறது. உருவமும் உள்ளடக்கமும் சார்ந்த உறுப்புக்கள் இணைவுபெறும் பொழுதில் "கலை முழுமை" அடைகிறது.

மார்க்சியத்தின் உருவம் - உள்ளடக்கம், அடித்தளம், மேற்கட்டுமானம், பிரதிபலித்தல் கொள்கை, சோஷலிச யதார்த்தவாதம் ஆகிய அழகியல் கொள்கைகள் கலை, இலக்கியத்தில் இடம் பெறுவதே மார்க்சிய இலக்கியக் கோட்பாடு. படைப்புகளிலும் படைப்புகள் குறித்த ஆராய்ச்சிகளிலும் இக்கோட்பாடுகள் அடிப்படைகளாக விளங்குகின்றன" (Thiyagu, 2019). "மார்க்சியம் என்பது ஒரேவிதமானது இல்லை. பன்மைத்தன்மை உள்ளது. ஒன்று மார்க்ஸ், எங்கல்ஸ், லெனின், ஸ்டாலின், மாவோ என்று சேகுவராவுடன் செல்வது. இன்னொன்று மார்க்ஸ், எங்கல்ஸ், பிளாக்கானோவ், லெனின், கிராம்ஷி, அடர்னோ, அல்தூஸர், பூகோ, லியோடார்ட் என்று செல்வது. முன்னது அரசியல் சார்ந்து அதிகாரம் நோக்கியது. பின்னது சிந்தனையின் ஊடாக மனித விடுதலையை நோக்கியது. அரசியல் நோக்கிலான சிந்தனை எக்காலத்திலும் வளர்முகமாகத்தான் உள்ளது" ஆகிய மேற்கண்ட விளக்கங்களை இரா. காமராசு தமது ஆய்வு நூலில் சுட்டுகிறார்.

\section{வரலாற்றுச் சூழமைவு}

“வரலாற்றுச் சூழமைவு, முக்கியமாக மார்க்சியத் திறனாய்வில் அதிகம் கவனத்தைப் பெற்றுள்ளது. ஹோமர், எஸ்கிலஹ் முதற்கொண்டு ஷேக்ஸ்பியர், கதே, பால்ஜாக் வரையிலான பலரின் கருத்துகளையும் மார்க்சும் ஏங்கல்சும் அவ்வக்கால வரலாற்று சூழமைவுகளின் தளத்தில் வைத்தே மதிப்பிடுவதையும் அவற்றை அந்தக் காலங்களின் வெளிப்பாடுகளாகவும் குரல்களாகவும் கருதுவதையும் அவர்களின் நூல்களிலே காணலாம் என்பதாக விளக்குகிறார். (Natarajan, 2013)

\section{'சுரங்கம்' புதினத்தின் மார்க்சியவழி பொருத்தப்பாடு}

எஸ். தோதாத்ரி அவர்கள் "சோஷலிச யதார்த்தவாதத்தின் அடிப்படைகள்" எனும் தமது நூலில் குறிப்பிட்டதன் அடிப்படையில் பொருத்தப்பாட்டைக் காண்போம். உலக இலக்கிய அரங்கில் யதார்த்தவாதம் வளர்த்து வைத்திருந்த விதிமுறைகளை அடியொற்றியே சோஷலிச யதார்த்தவாதம் எழுந்தது (Kamarasu, 2016a).

\section{யதார்த்த வாதத்தில் காணப்படும் சமூக ஆய்வு முறை}

குசிபா. வின்' சுரங்கம் புதினம் முழுவதுமாக தொழிலையும் தொழிற்சங்கத்தையும் வளர்ப்பதாகவும், படிப்படியாக சமூகமாற்றம் கோரியுமாக இது பரவிக்கிடக்கிறது. கள அனுபவங்களிலிருந்து சமூக ஆய்வு மேற்கொண்டு படிப்பினை கற்று, அடுத்த கட்டத்திற்கு நகர்த்துகிறது. ஒடுக்கப்பட்ட முதல் போராட்டத்தில் சுரங்க முதலாளிகளின் கூலிப்படைகளால் தாக்கப்பட்டு அதிலிருந்து பாடம் கற்றுத் தெளிகிறான் பிகாஸ். சகதொழிலாளிகளிடமிருந்து எழுச்சி பெறும் ஒரு தலைமைப் பண்பாளனை அடையாளம் கண்டடைந்து மேலும் போராட்டத்திற்கு ஊக்குவிக்கிறது தொழிலாளி வர்க்கம். யதார்த்தமான சூழலுடன் 'அஜிட்ப்ராப்' (agitprop) கிளர்ச்சிப் பிரச்சாரம் இணையவே, கூடுதல் வலு கிடைக்கிறது. போராட்டம் வெற்றிபெறுகிறது. சூழல் கனியவே, சுரங்கத்தொழில் பொதுத்துறை வசமாகிறது. 
வகைப்பாடான பாத்திரப்படைப்பு

நிலக்கரிச் சுரங்கத்தின் தொழிலாளர்கள் அவலநிலைக்குக் காரணமான போக்குகளை உன்னிப்பாக ஆராய்ந்து அதைக் களைய எண்ணித் தட்டிக்கேட்ட பிகாஸ் சௌதரி தாக்குதலுக்கு உள்ளாகிறான். லாபநோக்கு மட்டுமே கொண்ட முதலாளிகள் தமது ஏஜன்டுகளான ' மைனிங் சர்தார்', 'மைனிங் ம்ன்சி ' மூலமாக தொழிலாளர்களைக் கசக்கிப்பிழிவது, சமூகத்தில் உயர்ந்த அந்தஸ்து வேண்டி தமது மாமனார் குடும்பத்தையே தனது மகள் நிச்சயதார்த்தத்துக்கு அழைக்காத முதலாளி திவேரி, பெண்சபலம் கொண்ட கணவனின் பலவீனத்தை எதிர்க்கத் துணிவற்ற அவன் மனைவி சகுந்தலா தேவி, குடிக்கு அடிமையாகும் தொழிலாளர்கள், கந்துவட்டியில் கொழித்த ஈட்டிக்காரர்கள் உள்ளிட்ட கதைமாந்தர்கள் என்று பல பாத்திரங்கள் இடம் பெறுகின்றன.

\section{சமூகத்தைப் புறவயமாக ஏற்றுக்கொள்ளுதல்}

தன்னை முன்னிலைப்படுத்தாது சமூகநலனை முன்நிறுத்தியதால் சுரங்கத்தைக் காக்க தன்னுயிரை மாய்த்துக்கொண்ட முகமறியாத தன்பாத் தொழிலாளியின் 'ஆன்மா பிகாஸ் சௌதரி மீது இறங்கி' தலைமைப்பண்பை ஊட்டுகிறது. எனவே, திருமண பந்தத்தையும் துறக்கிறான்.

\section{சமூக விமரிசனம் ஆழமாக இடம் பெறுதல்}

கொள்ளை லாபமீட்டும் முதலாளித்துவ அமைப்பின் மீதான உரையாடல் தொழிலாளர்கள் மத்தியிலும் விவசாயிகள் மத்தியிலும் கொந்தளிப்பையும் அச்சத்தையும் தோற்றுவிக்கிறது. தனியார் நிலக்கரிச் சுரங்கம் தோண்ட இழப்பீடு கிடைக்கும் என்ற நம்பிக்கையில் நிலம் தந்து ஏமாந்த விவசாயிகளையும் போராட்டத்தில் இணைப்பதன் தேவையை உணர்த்தும் தொழிற்சங்கத்தலைவர், தலையாட்டித் தொழிற்சங்கங்களுக்கு மாற்றாக, வர்க்க சமரசமின்றிப் போராடும் தலைமையின் அவசியம் உள்ளிட்ட அம்சங்கள் சமூக விமர்சனமாக விவாதங்களில் இடம் பெறுவதோடு முரண்பாடுகளைக் கூர்மையடைய வைக்கின்றன.

\section{உழைப்பின் உன்னதம்}

எல்லாவற்றுக்கும் மேலாக உழைப்பின் உன்னதம் வலியுறுத்தப்படுகிறது. கூலியைக் கூட ஏமாற்றிப் பிடித்தம் செய்து சுரண்டுவது முதலாளித்துவ நடைமுறை. கந்துவட்டிக்காரர்களும் சாராயக்கடைக்காரர்ளும் முதலாளிகளுக்கு மறைமுகமாக சேவைபுரிவது முதலாளித்துவ சுதந்திரத்தில் அனுமதிக்கப்படுகிறது. நியாயமான கூலிகேட்டு அணிதிரளாமல் பார்த்துக் கொள்வதில் இது முதலாளிகளுக்கு உதவுகிறது. சாராயம், கந்துவட்டியில் அவதியுறும் தொழிலாளர் வர்க்கம் இற்று அழிவதை முதலாளி வர்க்கம் வெறுமனே வேடிக்கை பார்க்கிறது. தன்னுயிரைத் திரணமாக மதித்து வளமான எதிர்காலத்திற்காக உழைப்பவர்கள் ஒருமுறையே சாகிறார்கள் அடிமைகள் அன்றாடம் சாகின்றனர் என்பதைத் தெளிவாக உணர்த்துகிறது.

\section{சமூகம் இயங்கியல் பொருள்முதல்வாத முறையில் ஆய்வு}

இயக்கவியல் பொருள்முதல்வாதம் இதில் ஆதாரசுருதியாகச் செயல்படுகிறது. எனவேதான், தாக்குதலுக்கு உள்ளான தொழிலாளர்கள் மீண்டும் பிகாஸ் சௌதரி தலைமையில் அணிதிரள்கிறார்கள். வர்க்க எதிர்ப்பைத் தாங்கிக்கொள்ள இயலாத நிர்வாகம் திரண்டு விடாமல் உளவறிய முயல்வதையும் அலட்சியப்படுத்திவிட்டு முன்னேறுகிறது தொழிலாள வர்க்கம்.

\section{வர்க்கப் போராட்டத்தின் தன்மை}

வர்க்கப் போராட்டம் முழுமையாகச் சித்தரிக்கப்படுகிறது. உழைப்பவனைச் சார்ந்து நிற்கும் போக்கு காணப்படுகிறது. இதனையொட்டிய வரலாற்று உணர்வும் மனிதநேயமும் சமூக உணர்வும் மற்ற இலக்கியங்களில் காணமுடியாத அளவிற்கு விரிவாக இதில் இடம்பெற்றிருப்பதைக் 
காணமுடிகிறது. 'சுரங்கம்' ுதினம் முுுவதுமே அனுபவ ரீதியான படிப்பினைகளைக்கற்றுக் கொண்டு தொழிலாள வர்க்கம் முன்னேறியதைக் காணலாம் (Chinnappa Bharathi, 2012). நிலக்கரிச் சுரங்க நாட்டுடைமை எவ்வாறு நிறைவேறியது? ஒன்றுபட்ட தொழிலாளர்கள் விவசாயிகளின் உருக்குப்போன்ற ஒற்றுமையினால் தான்!. அளவில் ஏற்படும் மாற்றம், குணத்தில் மாற்றத்தை ஏற்படுத்தும். அதேபோலக் குணத்தில் ஏற்படும் மாற்றம் அளவில் மாற்றத்தை ஏற்படுத்தும். குறைந்த தொழிலாளர்கள் எண்ணிக்கை முதலாளிகளுக்குச் சாதகமே. கூடுதல் எண்ணிக்கை இதர பகுதி தொழிலாளர்களையும் வேலை இழப்பு அச்சத்திலிருந்து விடுவிக்கிறது. 'ஒற்றுமைக்கான போராட்டம், போராட்டத்திற்கான ஒற்றுமை' என்பதை தொழிற்சங்க இயக்கம் இயங்கியல் அடிப்படையில் அணுகுவதால் முதல் தோல்வியிலிருந்து பெறும் படிப்பினை படிநிலைகளில் வெற்றியடைவதில் முடிகிறது.

\section{முடிவுரை}

நாவலின் காலகட்டம் 1975 வரைக்குமானது என்று ஆசிரியர் குசிபா. நாவலின் முன்னுரையில் குறிப்பிடுகிறார். எண்ணற்ற போராட்டங்கள் மூலம் தனியாரிடமிருந்து பொதுத்துறைக்கு சுரங்கத்தொழில் மாறியது இருந்தபின்னும் சுரண்டல் முறை தொடர்கிறது. சுரங்கத் தொழில் இப்போது மாஃபியாக்களின் பிடியில் சிக்கித் தவிக்கிறது. கொள்ளை லாபம் ஈட்டும் சுரங்கத்தொழில் மீண்டும் தனியாருக்குத் தாரை வார்க்கப்பட்டுவிட்டது. 1972- 73 ஆம் ஆண்டு தேசிய மயமாக்கப்பட்ட சுரங்கத்தொழில் சட்டங்கள், மார்ச் 2015- ஆம் ஆண்டு திரும்பப்பெறப்பட்டு மீண்டும் தனியாருக்குத் திறந்துவிடப்பட்டுள்ளது கொடும் சோகமானது. கரியற்ற சக்கையான சுரங்கங்கள் கைவிடப்பட்டு வேறு இடங்களை நாடுவதற்கு ஒப்பாக தொழிலாளர்களும் சக்கையானபின் தூக்கி வீசப்படுகிறார்கள். மீண்டும் பொதுத்துறைக்கு மாற்றப்பட்டு சுரண்டல் முறைக்கு முடிவுகட்டும் வரை விடியல் இல்லை. உலகமயமாக்கல் காலகட்டத்தில் 'மீண்டும் லாபம், அதிக லாபம்' மட்டுமே குறிக்கோள் சுரண்டல் புதுப்புது வடிவங்களில் உருவாகிறது. பன்னாட்டுப் பெருவணிக வல்லூறுகள் சுரங்கங்களின் பள்ளத்தாக்குகள் மீது வட்டமிடுகின்றன. உலகளாவிய வலதுசாரி அரசுகளின் எழுச்சியால் இடதுசாரித் தொழிற்சங்கங்கள் குறிவைத்து தாக்கப்படுகின்றன. ஆனாலும் சுரண்டல் அமைப்பு நீடிக்கும் வரை போராட்டமும் புதுவீச்சுடன் ம்ன்னேறும். "தன்பாத் சுரங்கத்தில் ஒரரு தொழிலாளி பிறருக்காக எப்படி தன்னைத்தானே உயிர்ப்பலி கொடுத்துக் கொண்டான்? "என்ற தொழிலாளர்களின் அடிமனதில் உறைந்துவிட்ட சித்திரம் வீரயுக எழுச்சிப்பாடலாக ஒலிக்கும். பிகாஸ் (விகாஸ்) என்னும் வங்கமொழிப் பதத்திற்குப் பல பொருள் உண்டு: வெளிச்சம், பிரகாசம், வளர்ச்சி என்று. சுரங்க வாயிலில் வெளிச்சம் பரவி நிரந்தர விடியல் காணும். சுரங்கம் புதினத்தின் வெற்றி என்பது வேற்று மொழிப்பகுதியில் கள ஆய்வு செய்து தமிழில் எழுதி மீண்டும் அந்தந்த வட்டார மொழிகளில் மொழிபெயர்க்கப்பட்டு உணர்வூட்டுவது என்பதில் அடங்கியுள்ளது. இதுபோல் கள ஆய்வுகள் மேற்கொள்ளப்படுவது தாய்மொழி தமிழுக்கும், தமிழ்ப்படைப்பாளிகளுக்கும் பெருமை சேர்க்கும். அத்தகைய படைப்புகளை வரலாற்றுச் சூழமைவு என்னும் வரலாற்றின் அடிப்படையில் நோக்குதலுக்கு மார்க்சியர்கள் மதிப்பீடு செய்துள்ளதை நினைவில் கொள்ள வேண்டும்.

\section{References}

Chinnappa Bharathi, Ku., (2012) Surangam Novel, Bharathi Puthakalayam, Chennai, India.

Kamarasu, Ra., (2016a) Marxism, tamil sitrithalgal vali uruvana naveena thiranaivu pokukal, Tamil University, Chennai, India.

Kamarasu, Ra., (2016b) Yathaarththavatham, Tamil Sitrithalgal Vali Uruvana Naveena Thiranaivu Pokukal, Tamil University, Chennai, India.

Natarajan, T, S., (2013) Thiranaivu Kalai Kolkaigalum Anugumuraigalum, New Century Book House, Chennai, India.

Rius, (2017) Karl Marx Sithira Kathaigal, Vidiyal Pathipagam, Chennai, India.

Thiyagu, (2019) Maarksiyam Ana Aavanaa, Tamil desam veliyedu, Chennai, India. 
Funding: No funding was received for conducting this study.

Conflict of Interest: The Author has no conflicts of interest to declare that they are relevant to the content of this article.

About the License:

\section{(1)(1)}

Attribution 4.0 International (CC BY 4.0)
(C) The Author 2022. The text of this article is open access and licensed under a Creative Commons Attribution 4.0 International License 